Published in final edited form as:

J Dent Res. 2006 April ; 85(4): 313-317.

\title{
Cigarette Smoking Increases the Risk of Root Canal Treatment
}

\author{
E.A. Krall ${ }^{1,2,{ }^{*} \text {, C. Abreu Sosa }}{ }^{3}$, C. Garcia ${ }^{2}$, M.E. Nunn${ }^{2}$, D.J. Caplan ${ }^{4}$, and R.I. Garcia ${ }^{1,2}$ \\ 1 VA Dental Longitudinal Study, VA Boston Healthcare System \\ 2Department of Health Policy \& Health Services Research, Boston University Goldman School of Dental \\ Medicine, 715 Albany Street, 560, Room 338, Boston, MA 02118, USA
}

3University of Puerto Rico School of Dentistry

4Department of Dental Ecology, University of North Carolina School of Dentistry

\begin{abstract}
Few studies have investigated smoking as a risk factor for root canal treatment. We studied the effect of smoking on the incidence of root canal treatment, controlling for recognized risk factors, in 811 dentate male participants in the VA Dental Longitudinal Study. Participants were not VA patients. Follow-up ranged from 2 to 28 years. Root canal treatment was verified on radiographs and evaluated with proportional hazards regression models. Compared with never-smokers, current cigarette smokers were 1.7 times as likely to have root canal treatment $(\mathrm{p}<0.001)$, but cigar and/or pipe use was not significantly associated with root canal treatment. The risk among cigarette smokers increased with more years of exposure and decreased with length of abstinence. These findings suggest that there is a dose-response relationship between cigarette smoking and the risk of root canal treatment.
\end{abstract}

\section{Keywords}

smoking; tobacco; endodontics; root canal treatment

\section{INTRODUCTION}

Root canal treatment is often indicated when the dental pulp becomes infected by bacteria. Caries lesions, dental procedures, and tooth fracture increase the likelihood of bacterial access. Factors that alter the host response to inflammation, such as smoking, may also indirectly influence the risk of infection and its subsequent treatment. Little research has been conducted on the association between smoking and root canal treatment. Bergström et al. (2004) reported that current smokers had a higher prevalence of endodontic treatment and a higher percentage of treated roots than did non-smokers, but, after adjustment for age, the differences were not statistically significant. An important limitation of that study was its cross-sectional design (Garcia, 2005).

Smoking impairs the body's responses to infection (Das, 1985; Tracy et al., 1997; Frohlich et al., 2003), exacerbates bone loss in the systemic skeleton (Krall and Dawson-Hughes, 1991) and oral cavity (Krall et al., 1999), decreases the blood's oxygen-carrying capacity (Ijzerman et al., 2003), and causes vascular dysfunction (Pittilo, 1990). Any one of these pathophysiologic pathways can potentially affect the health of the tooth pulp and surrounding bone tissues and result in a higher incidence of root canal treatment in smokers than in non-smokers. We

*corresponding author, kralle@bu.edu. 
investigated this hypothesis in a cohort of adult men who have been followed for three decades in the VA Dental Longitudinal Study.

\section{MATERIALS \& METHODS}

\section{Subjects}

The VA Dental Longitudinal Study (DLS) is a closed-panel prospective study of oral health and aging in men (Kapur et al., 1972). At the study baseline in 1968-73, the DLS enrolled 1231 medically healthy men, ages 21 to 84 yrs, who concurrently participated in the Normative Aging Study (Bell et al., 1966). The men were not patients of the VA healthcare system, but received dental and medical care from the private sector. Participants returned to the study site every three years to receive clinical and radiographic dental examinations and complete questionnaires (see APPENDIX). Up to 28 yrs of follow-up are included in this analysis. The study was reviewed and approved by the Department of Veterans Affairs Subcommittee on Human Studies and the Boston University Medical Center Institutional Review Board. All participants gave written informed consent.

\section{Examinations}

At each examination, the number of teeth remaining was counted, and each tooth was evaluated for restorations, caries, periodontal probing at six sites, and clinical assessments of tooth mobility and calculus. Probing pocket depth, mobility, and calculus were recorded as ordinal scores. Pocket depth scores ranged from $0(\leq 2 \mathrm{~mm})$ to $3(\geq 5 \mathrm{~mm})$; mobility scores from 0 (none) to 4 (depressability); and calculus scores from 0 (none) to 3 (circumferential band around tooth). Alveolar bone loss was measured from periapical radiographs on the distal and mesial sites of each tooth by a modified Schei ruler method (Schei et al., 1959), which expresses the reduction in alveolar bone height as the percent of the total distance between the cementoenamel junction and the root apex. The maximum probing depth and bone loss scores per tooth were used in analyses. Radiographic caries was also assessed.

Of the 1231 men initially enrolled in the DLS, 1058 were potentially eligible for this analysis, on the basis of having 2 or more teeth at baseline. Over a period of $1 \mathrm{yr}$, two Boston University endodontic residents, each trained and calibrated, reviewed serial periapical radiographs from a randomly ordered sample of those eligible $(n=852)$ to determine the presence or absence of root canal treatment and periradicular radiolucency. The final dataset consisted of 811 men with at least one follow-up examination and complete smoking information, and 18,893 teeth that were free of root canal treatment at baseline. Because the exact date of root canal treatment was not known, the date of onset was systematically computed as the midpoint between the DLS examination date at which the root canal treatment first appeared on radiograph and the examination date that immediately preceded it. The endodontists each independently reviewed 576 radiographs, and the kappa for inter-examiner agreement for the presence/absence of root canal treatment was 1.00 .

Education, frequency of toothbrushing and flossing, and smoking history were obtained by interviewer-administered questionnaires. At baseline, cigarette status and duration were assessed by these questions: "Have you ever smoked cigarettes?", "Have you smoked in the last year?", "How long have you been smoking cigarettes?", "How long has it been since you quit smoking?", and "For how many years were you a cigarette smoker?" Smoking duration and time since quitting prior to baseline were asked only at baseline. Cigarette status and dose were updated, at each follow-up examination between 1971 and 1985, with "Do you use tobacco?" and "If yes, how many packs of cigarettes per day?". The questions were changed to "Do you smoke at all?", "If yes, do you smoke cigarettes?", and "How many cigarettes in 
an average day $\left(1 / 2,1,1 \frac{1}{2}\right.$, or $2+$ packs/day)" at follow-up examinations after 1985 . Similar questions at all examinations assessed cigar- and pipe-smoking information.

\section{Statistical Analysis}

Participants were categorized on the basis of smoking status during follow-up: never-smoker, former cigarette smoker (quit prior to the DLS baseline examination), current cigarette smoker (for any part of follow-up), or current cigar and/or pipe smoker. Men who smoked cigarettes in combination with either pipes or cigars were classified as cigarette smokers.

Characteristics of the men by smoking status were compared with parametric or non-parametric analysis of variance (continuous variables) or chi-square statistic (categorical variables). In participant-level analyses, risk of any incident root canal treatment was estimated with proportional hazards regression. Current cigarette- and cigar/pipe-smoking status at each examination was treated as a time-dependent covariate. In addition, the risks of incident root canal treatment by current cigarette status, number of years smoked, and number of years since last smoked were estimated at the tooth level with multivariate proportional hazards regression to account for multiple root canal treatments within individual participants. All analyses were performed with never-smokers $(\mathrm{n}=230)$ as the reference group, and again with never and former cigarette smokers $(n=440)$ as the reference group. Since the results were not substantially different, only the risks relative to never-smokers are presented. Differences were considered statistically significant at $\mathrm{p}<0.05$. Hazards ratios (HR) and $95 \%$ confidence intervals $(95 \% \mathrm{CI})$ are presented.

\section{RESULTS}

The participants were $48 \pm 9$ (mean \pm SD) yrs old at baseline and had $24 \pm 6$ teeth remaining. Table 1 shows characteristics by smoking status. Current cigarette smokers were younger, had fewer teeth remaining, the highest percentages of teeth with calculus, alveolar bone loss $>20 \%$, and probing pocket depth $>3 \mathrm{~mm}$, and had lost more teeth than never-smokers, former cigarette smokers, or current cigar/pipe smokers.

\section{Incidence of Root Canal Treatment}

Incident root canal treatments were observed in 998 teeth among 385 participants. Teeth that had incident root canal treatment were more likely, at the examination prior to root canal treatment, to be crowned ( $21 \mathrm{vs} .4 \%)$, have periradicular radiolucency ( $8 \mathrm{vs.} 0 \%)$, mobility $\geq$ $0.5 \mathrm{~mm}$ ( 5 vs. $2 \%)$, alveolar bone loss > 20\% (27vs. 12\%), probing pocket depth $>3 \mathrm{~mm}$ ( 28 vs. $16 \%$ ), any coronal caries ( $30 v s .19 \%$ ), any fillings ( $75 v s .56 \%$ ), and fillings on all 5 surfaces $(5 v s .2 \%)$ relative to teeth that were never treated (all $\mathrm{p}<0.001)$.

The age-adjusted risk of incident root canal treatment was significantly greater in current cigarette smokers, relative to never-smokers, but not in cigar or pipe smokers (Table 2). The hazards ratio attributable to cigarette smoking was similar, but remained statistically significant, in models that also controlled for number of teeth present, any teeth with periradicular radiolucency, and percentages of teeth with crowns, coronal caries, and alveolar bone loss $>20 \%$. The results were not changed when education, brushing, or flossing was included in the models. Multivariate analyses of root canal treatment risk at the tooth level yielded similar hazards ratios when controlled for age, presence of a crown, any coronal caries, radiolucency, and alveolar bone loss level (HR and 95\% CI for current cigarette use $=1.9,1.4$ to 2.5 ; for current cigar/pipe use $=1.3,0.9$ to 1.7 ).

The association of root canal treatment risk varies with length of time since last smoked (Fig 1). Relative to never-smokers, the hazards ratio was elevated for the teeth of men who had quit 
9 or fewer years prior to baseline ( $\mathrm{HR}=1.9,95 \% \mathrm{CI}=1.3$ to 2.8 ). But among men who had quit more than 9 yrs previously, the hazards ratio was similar to that in never-smokers ( $\mathrm{HR}=$ $1.0,95 \% \mathrm{CI}=0.7$ to 1.5$)$.

Among current cigarette smokers, the hazards ratio for root canal treatment increased from 1.2 $(95 \% \mathrm{CI}=0.7$ to 1.9$)$ for teeth in men who smoked a total of $\leq 4 \mathrm{yrs}$, to $2.0(95 \% \mathrm{CI}=1.2$ to

$3.3)$ in those who smoked from 5 to $12 \mathrm{yrs}$, and $2.2(95 \% \mathrm{CI}=1.5$ to 3.3$)$ in those who smoked $>12$ yrs (Fig. 2).

\section{DISCUSSION}

To our knowledge, this is the first longitudinal analysis that describes smoking as a risk factor for root canal treatment. The outcome in our study, root-canal-treated teeth, is a surrogate for periapical periodontitis and underestimates the true incidence of such lesions. Caplan (2004) reviewed community-based studies of periapical periodontitis and root canal treatment and found the prevalence of non-root-filled teeth with periapical periodontitis to be low (from 0.1 to 5\%). Similarly, Bergström et al. (2004) reported that a small percentage, approximately $1-3 \%$, of teeth had untreated lesions. However, a more important consideration is that root canal treatment is only one possible outcome of teeth with periapical periodontitis; another is tooth extraction, which is more prevalent in smokers than in non-smokers and results in an unknown proportion of potentially root-canal-treated teeth being lost to follow-up.

Conclusions from the few cross-sectional studies on smoking and periapical periodontitis are inconsistent. Bergström et al. (2004), in a study of 241 individuals, found no evidence that smoking increased the risk of periapical lesions. Although the prevalence of periapical periodontitis was higher in current and former smokers than in non-smokers, the unadjusted odds ratio (1.6) was not statistically significant and was further reduced in multiple logistic regression analyses. However, the authors focused on current endodontic disease and did not include past treatment as an outcome (Garcia, 2005). Smokers had 30\% more endodontically treated roots, but the difference was not significant after adjustment for age. In a study of 613 individuals, Kirkevang and Wenzel (2003) also reported an odds ratio of 1.6 for smoking and periapical periodontitis, which was significant in multiple logistic regression models.

The most common means of bacterial entry to the pulp cavity are caries lesions on the coronal or root surfaces. Secondary caries, inadequate coronal restorations, and root caries were each associated with increased odds of periapical periodontitis (Kirkevang and Wenzel, 2003). In our study, adequacy of restoration was not assessed, and root caries was not noted separately in the first several examination cycles, but crowns and coronal caries each increased the risk of root canal treatment. Prospective studies suggest that smokers tend to have more decayed or filled coronal (Drake et al., 1997) and root surfaces (Locker, 1996) than do non-smokers.

Smokers have impaired responses to infection. The functions of polymorphonuclear leukocytes, macrophages, T-cell lymphocytes, antibodies, and immunoglobulins A, G, and M are suppressed in smokers (Holt, 1987). Smokers have elevated levels of mediators of acutephase inflammatory response, such as TNF- $\alpha$ (Tappia et al., 1995) and IL-6 (de Maat and Kluft, 2002), that are capable of inflicting damage to tissues. Smoking also induces a chronic systemic inflammatory response that may persist even after smoking has actively ceased. C-reactive protein (CRP) levels in serum were higher in current smokers than in non-smokers (Frohlich et al., 2003), particularly among heavy smokers of more than 1 pack per day (Das, 1985). In addition, CRP levels were greatest among those with longer duration of smoking and more pack-years, and were inversely correlated with duration of abstinence (Frohlich et al., 2003). Peripheral leukocyte counts are also higher in current smokers than in non-smokers and exhibit dose-response relationships (Sparrow et al., 1984; Schwartz and Weiss, 1991; Tracy et al., 
1997), although, as noted above, their function is impaired. These observations suggest that once bacterial infection begins in the pulp and surrounding tissues, smokers are less likely than non-smokers to be able to limit the destruction.

Another underlying explanation for our findings is impaired oxygen delivery and damage to the circulatory system inside the tooth. Levels of carboxyhemoglobin in the blood are elevated in smokers, resulting in a decreased oxygen-carrying capability. In addition, smoking is associated with impaired microvascular function and endothelial cell injury in blood vessels, possibly due to free-radicals found in tobacco smoke (Ijzerman et al., 2003). Blood vessels in the healthy gingiva constrict in reaction to environmental agents that induce stress, a response identical to that in the extremities (Giddon, 1966; Zelechowski et al., 1969). Because components in cigarette smoke induce stress (Giddon, 1966) and reduce blood flow volume, vessels that serve the tooth root are likely to show signs of vascular dysfunction that restrict nutrient supply and impede cellular repair. This suggests that smoking may be a factor that contributes to early tissue death within the pulp cavity.

There may also be residual confounding factors that contribute to the increased root canal treatment risk we observed in current cigarette smokers. Smoking is inversely correlated with socio-economic status and may be a marker for poor dental hygiene or lack of dental care (USDHHS, 2000). In our study, current cigarette smokers tended to have less education, although adjustment for this characteristic did not change the risk estimates. There may be differences between smokers and non-smokers in addition to oral hygiene practices and education that we were unable to measure adequately, but that could further attenuate the observed association between smoking and root canal treatment.

Cigar and pipe use is related to periodontal disease (Krall et al., 1999) and tooth loss (Krall et al., 1997) in this cohort, but not to root canal treatment risk. This finding may be due to the small number of cigar and pipe smokers, or to our difficulty in quantifying the amount smoked. Among cigarette smokers, risk estimates suggested a threshold effect around 5 yrs of exposure. Since cigar smokers tend to initiate the habit at a later age than do cigarette smokers (Gerlach et al., 1998), their total tobacco exposure may be limited. It is possible that a relationship exists between root canal treatment and cigars and pipes, but we did not have a sufficiently large sample size or exposure high enough to detect it.

Another limitation of the study is the gender and ethnic composition of the participant population. The DLS is a closed-panel prospective study that did not include women at its inception and has less than 5\% minority participants. We would expect to see an association between smoking and root canal treatment risk in women and other ethnic groups as well, although absolute risk levels may differ and might be more closely related to cigarette exposure than to demographic characteristics.

Important strengths of the current study that differentiate it from previous studies are the large number of participants, the prospective design with long follow-up time, the frequent intervals at which radiographs were taken, control for caries and restorations, and the findings of doseresponse relationships with years of exposure and duration of abstinence. The results suggest that smoking may have a causal role in the development of lesions that result in root canal treatment, and, conversely, that smoking prevention and cessation are critical strategies to reducing the risk of root canal treatment.

\section{Supplementary Material}

Refer to Web version on PubMed Central for supplementary material. 


\section{Acknowledgements}

This study was supported by a US Dept. of Veterans Affairs Epidemiology Merit Review grant and by the Massachusetts Veterans Epidemiology Research and Information Center, and by NIH grants R01 DE 013807, T35 DE07268, R01 DA10073, R15 DE12644, M01 RR 000533, and K24 DE00419. We thank Dr. Joel Chasen and Dr. Ryan Yamanaka for data collection on endodontic treatment status. Parts of the information in this manuscript were presented at the 2004 annual meeting of the Society for Research on Nicotine and Tobacco, Scottsdale, AZ, February, 2004.

\section{References}

Bell B, Rose CL, Damon A. The Veterans Administration longitudinal study of healthy aging. Gerontologist 1966;6:179-184. [PubMed: 5342911]

Bergström J, Babcan J, Eliasson S. Tobacco smoking and dental periapical condition. Eur J Oral Sci 2004;112:115-120. [PubMed: 15056107]

Caplan DJ. Epidemiologic issues in studies of association between apical periodontitis and systemic health. Endodont Top 2004;8:15-35.

Das I. Raised C-reactive protein levels in serum from smokers. Clin Chim Acta 1985;153:9-13. [PubMed: 2416495]

de Maat MP, Kluft C. The association between inflammation markers, coronary artery disease and smoking. Vascul Pharmacol 2002;39:137-139. [PubMed: 12616981]

Drake CW, Beck JD, Lawrence HP, Koch GG. Three-year coronal caries incidence and risk factors in North Carolina elderly. Caries Res 1997;31:1-7. [PubMed: 8955986]

Frohlich M, Sund M, Lowel H, Imhof A, Hoffmeister A, Koenig W. Independent association of various smoking characteristics with markers of systemic inflammation in men. Results from a representative sample of the general population (MONICA Augsburg Survey 1994/95). Eur Heart J 2003;24:13651372. [PubMed: 12871694]

Garcia RI. Commentary on tobacco smoking not found to be related to apical periodontitis. J Evid Base Dent Pract 2005;5:90-91.

Gerlach, KK.; Cummings, KM.; Hyland, A.; Gilpin, EA.; Johnson, MD.; Pierce, JP. Trends in cigar consumption and smoking prevalence. In: Burns, D.; Cummings, KM.; Hoffmann, D., editors. Cigars: health effects and trends. Monogr. No. 9. NIH Publication 98-4302. Bethesda, MD: National Institutes of Health, National Cancer Institute; 1998.

Giddon DB. Psychophysiology of the oral cavity. J Dent Res 1966;45:1627-1636. [PubMed: 5226640]

Holt PG. Immune and inflammatory function in cigarette smokers. Thorax 1987;42:241-249. [PubMed: 3303428]

Ijzerman RG, Serne EH, van Weissenbruch MM, de Jongh RT, Stehouwer CD. Cigarette smoking is associated with an acute impairment of microvascular function in humans. Clin Sci (Lond) 2003;104:247-252. [PubMed: 12605581]

Kapur KK, Glass RL, Loftus ER, Alman JE, Feller RP. The Veterans Administration longitudinal study of oral health and disease. Aging Hum Devel 1972;3:125-137.

Kirkevang LL, Wenzel A. Risk indicators for apical periodontitis. Community Dent Oral Epidemiol 2003;31:59-67. [PubMed: 12542433]

Krall EA, Dawson-Hughes B. Smoking and bone loss among postmenopausal women. J Bone Miner Res 1991;6:331-338. [PubMed: 1858519]

Krall EA, Dawson-Hughes B, Garvey AJ, Garcia RI. Smoking, smoking cessation, and tooth loss. J Dent Res 1997;76:1653-1659. [PubMed: 9326897]

Krall EA, Garvey AJ, Garcia RI. Alveolar bone loss and tooth loss in male cigar and pipe smokers. J Am Dent Assoc 1999;130:57-64. [PubMed: 9919032]

Locker D. Incidence of root caries in an older Canadian population. Community Dent Oral Epidemiol 1996;24:403-407. [PubMed: 9007358]

Pittilo RM. Cigarette smoking and endothelial injury: a review. Adv Exp Med Biol 1990;273:61-78. [PubMed: 2288292]

Schei O, Waerhaug J, Lovdal A, Arno A. Alveolar bone loss as related to oral hygiene and age. J Periodontol 1959;30:7-16. 
Schwartz J, Weiss ST. Host and environmental factors influencing the peripheral blood leukocyte count. Am J Epidemiol 1991;134:1402-1409. [PubMed: 1776614]

Sparrow D, Glynn RJ, Cohen M, Weiss ST. The relationship of the peripheral leukocyte count and cigarette smoking to pulmonary function among adult men. Chest 1984;86:383-386. [PubMed: 6467999]

Tappia PS, Troughton KL, Langley-Evans SC, Grimble RF. Cigarette smoking influences cytokine production and antioxidant defences. Clin Sci (Lond) 1995;88:485-489. [PubMed: 7540525]

Tracy RP, Psaty BM, Macy E, Bovill EG, Cushman M, Cornell ES, et al. Lifetime smoking exposure affects the association of C-reactive protein with cardiovascular disease risk factors and subclinical disease in healthy elderly subjects. Arterioscler Thromb Vasc Biol 1997;17:2167-2176. [PubMed: 9351386]

US Department of Health and Human Services. Understanding and improving health and objectives for improving health. 2nd. 1, 2. Washington, DC: US Government Printing Office; 2000. Healthy People 2010.

Zelechowski K, Giddon DB, Clark RE. Gingival vascular response to anticipated and actual punctate stimulation. J Dent Res 1969;48:419-422. [PubMed: 5254482] 


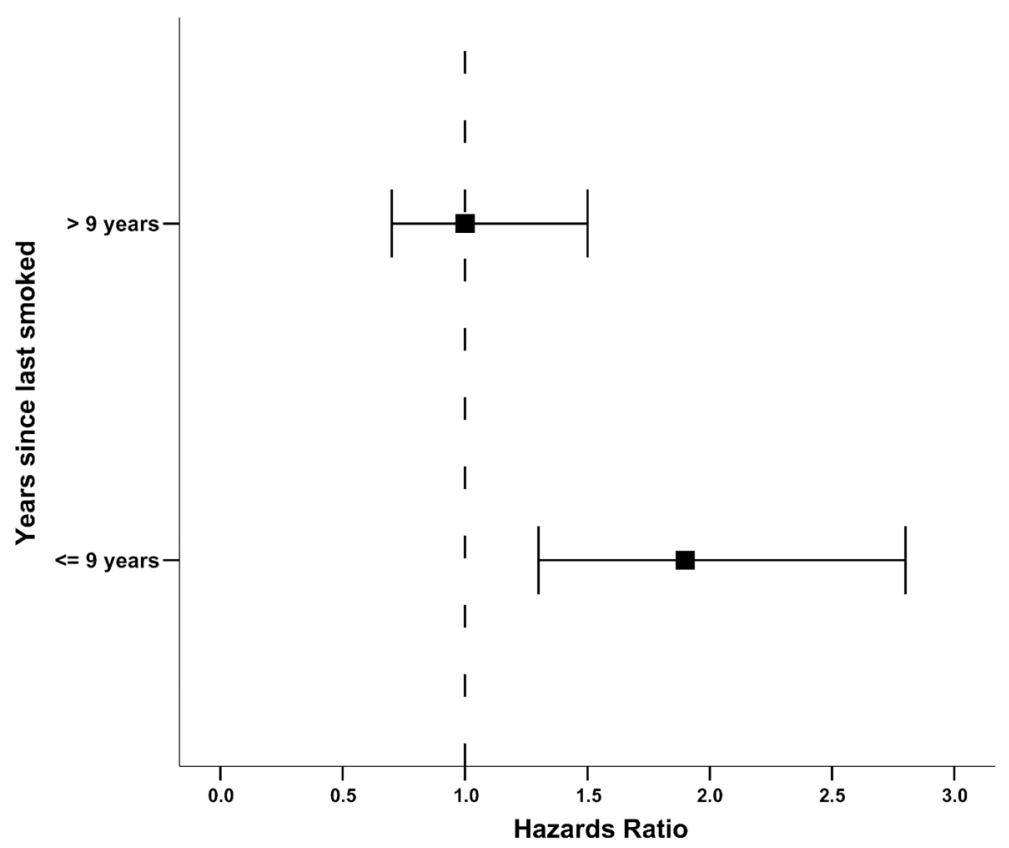

Figure 1.

Hazards ratios ( $\mathbf{a})$ and $95 \%$ confidence intervals (error bars) for risk of root canal treatment among former cigarette smokers (smoked prior to study baseline, but remained abstinent during follow-up), by length of time since last smoked. The median time since last smoked was 9 yrs. Numbers in each interval are 111 participants/2587 teeth in the $\leq 9$-year group and 99 participants/2312 teeth in the $>9$-year group. Never-smokers $(n=230$ participants $/ 5765$ teeth) are the reference group. HR are adjusted for age, presence of crown (yes, no), any caries (yes, no), periradicular radiolucency (yes, no), and alveolar bone loss score. 


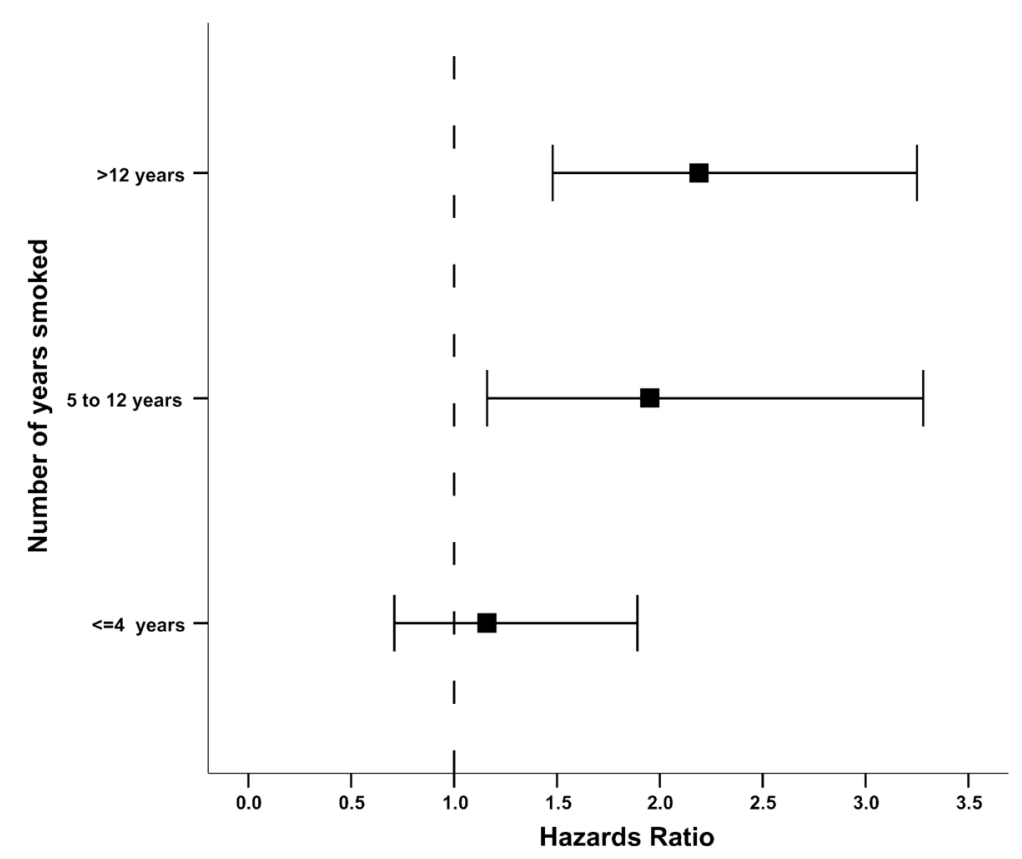

Figure 2.

Hazards ratios ( $\mathbf{a})$ and $95 \%$ confidence interval (error bars) for risk of root canal treatment among current cigarette smokers, by total yrs smoked. Numbers in each interval are 48 participants/ 1347 teeth with $\leq 4$ yrs, 78 participants/1782 teeth with 5 to 12 yrs, and 99 participants/2099 teeth with $>12$ yrs. Never-smokers $(n=230$ participants/5765 teeth $)$ are the reference group. Hazard ratios are adjusted for age, presence of crown (yes, no), any caries (yes, no), periradicular radiolucency (yes, no), and alveolar bone loss score. 


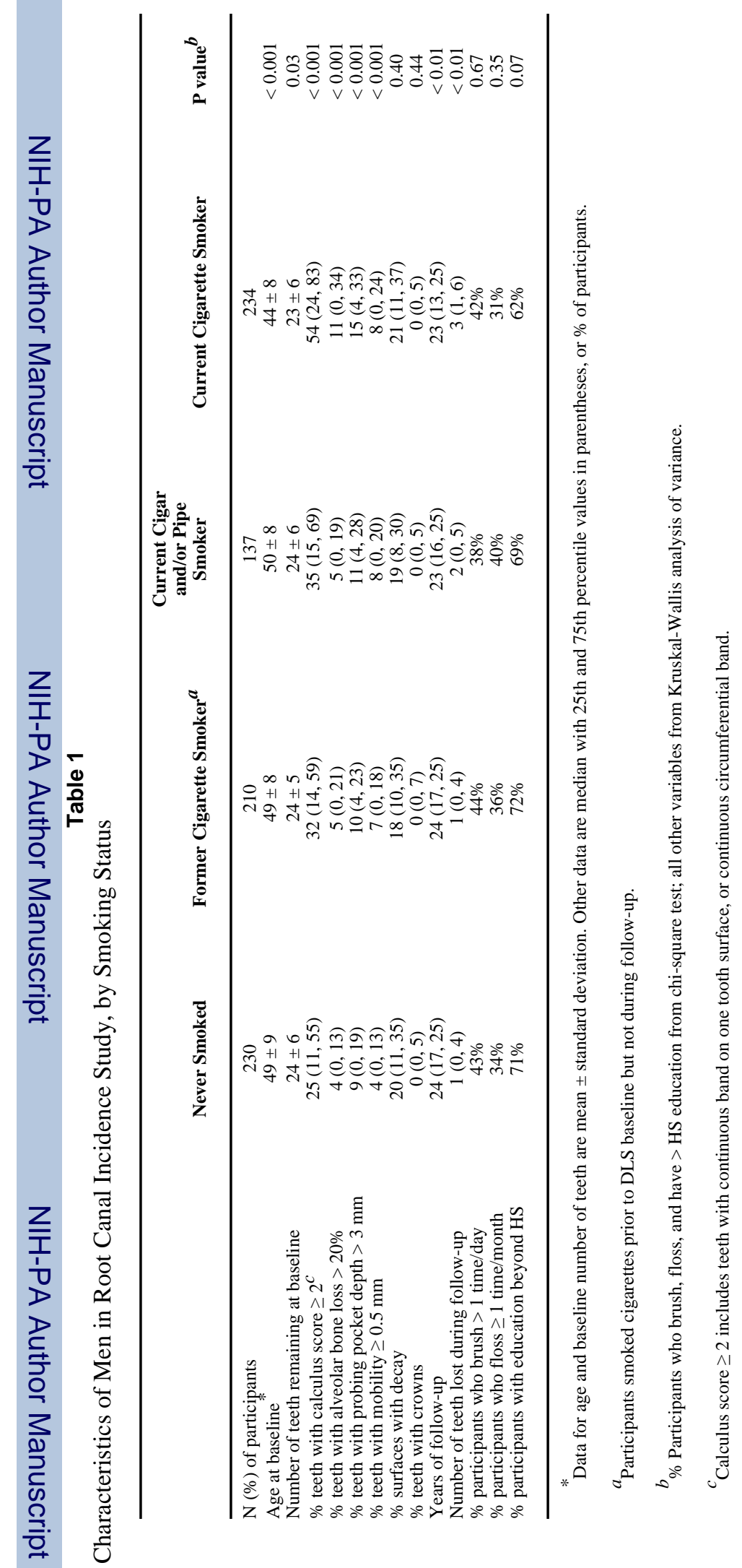


Table 2

Smoking Status and the Risk of Root Canal Treatment Relative to Never-smokers: Hazards Ratios and 95\% Confidence Intervals for Cigarette Smokers and Cigar/Pipe Smokers Relative to Never-smokers

\begin{tabular}{lcc}
\hline Model and Independent Variables & Current Cigarette Use & Current Cigar/Pipe Use \\
\hline Age & $1.7(1.3-2.3)$ & $1.9(1.4-2.6)$ \\
Age, \# baseline teeth, \% teeth with caries, \% teeth with crowns, any & $1.8-1.5)$ \\
teeth with periradicular radiolucency & $1.7(1.2-2.3)$ & $1.3(0.9-1.8)$ \\
Same as model 2, plus \% teeth with alveolar bone loss $>20 \%$ & $1.2(0.9-1.8)$ \\
\hline
\end{tabular}

